[0212-7199 (2005) 22: 3; pp 130-132] ANALES DE MEDICINA INTERNA Copyright (C) 2005 ARAN EDICIONES, S.L

AN. MED. InTERnA (Madrid) Vol. 22, N. ${ }^{\circ}$ 3, pp. 130-132, 2005

\title{
Deterioro de la capacidad funcional en pacientes ancianos ingresados en un Servicio de Medicina Interna
}

\author{
M. D. MAÑAS, E. MARCHÁN, C. CONDE, S. SÁNCHEZ, T. SÁNCHEZ-MAROTO, \\ M. C. MOLINA
}

Servicio de Medicina Interna. Complejo Hospitalario de Ciudad Real. Ciudad Real

\begin{abstract}
FUNCTIONAL IMPAIRMENT IN ELDERLY PATIENTS HOSPITALISED IN AN INTERNAL MEDICINE UNIT
\end{abstract}

\section{RESUMEN}

Valoramos la repercusión en la capacidad funcional de los pacientes ancianos tras un ingreso hospitalario en un servicio de Medicina Interna.

Se estudió de forma prospectiva los pacientes con 80 años o más ingresados en el Servicio de Medicina Interna del Hospital Provincial de Ciudad Real, entre los meses de febrero a julio de 2003. La valoración de la capacidad funcional se realizó mediante el índice de Barthel.

Se incluyeron $206(77,4 \%)$ con un índice de Barthel previo de 70,9, al ingreso de 48,9 y al alta de 58,6 ( $<<0,001)$. Se recogió un índice de Barthel al alta menor al previo en el 73,8\% de los pacientes.

La hospitalización conlleva un deterioro importante en la capacidad funcional de los pacientes ancianos. Es necesario identificar los factores de riesgo responsables de esta pérdida funcional y poder establecer pautas preventivas de actuación.

PALABRAS CLAVE: Capacidad funcional. Índice de Barthel. Paciente anciano. Hospitalización.

\begin{abstract}
To determine the functional status in elderly patients after a hospitalisation in an Internal Medicine unit.

We prospectively studied patients aged 80 or above hospitalised in the Hospital Provincial de Ciudad Real in an Internal Medicine unit, between February and July, 2003. The functional status was determined by Barthel Index.

We examined 206 patients (77,4\%). They showed a previous Barthel Index of 70,9; one of 48,9 in the hospitalisation stage and one of 58,6 when discharged $(p<0,001)$. We noticed a Barthel Index when discharged which was lower than the previous Barthel one in 73,8\% patients.

Hospitalisation implies a great functional impairment in the functional status elderly patients. It would be convenient, therefore, to identify the risk factors to be able to set some guidelines for a preventive model.
\end{abstract}

KEY WORDS: Functional impairment. Barthel index. Elderly patients. Hospitalisation.

Mañas MD, Marchán E, Conde C, Sánchez S, Sánchez-Maroto T, Molina MC. Deterioro de la capacidad funcional en pacientes ancianos ingresados en un servicio de Medicina Interna. An Med Interna (Madrid) 2005; 22: 130-132.

\section{INTRODUCCIÓN}

La valoración de la situación funcional en el anciano permite identificar su nivel de independencia para realizar las actividades básicas de la vida diaria (ABVD) y las actividades instrumentales de la vida diaria(AIVD). Esta valoración se puede realizar mediante la utilización de escalas, como el índice de Barthel (1,2). Fue desarrollado por Mahoney y Barthel en el Hospital de crónicos de Maryland para evaluar los progresos en la independencia de pacientes con patología neuromuscular y/ó musculoesquelética (3).

En diversos estudios se describe una incidencia de deterioro funcional mayor del $70 \%$ en el momento del ingreso y en torno al $30 \%$ en el momento del alta (4-6). A pesar de la importancia del problema, son relativamente escasos los estudios que analizan el deterioro funcional del paciente anciano como consecuencia del ingreso en una unidad de agudos (4-14).

El objetivo principal del presente trabajo es valorar la repercusión de la hospitalización en la capacidad funcional de los pacientes ancianos ingresados en nuestra área, utilizando el índice de Barthel.

\section{CASOS APORTADOS}

Estudio prospectivo de todos los pacientes con 80 o más años ingresados en el Servicio de Medicina Interna del Hospital Provin-

Trabajo aceptado: 14 de octubre de 2004 
cial de Ciudad Real, entre el 20 de febrero y el 1 de julio de 2003. El servicio dispone de 28 camas para la hospitalización de pacientes mayores de 75 años no subsidiarios de cuidados intensivos ni realización de técnicas invasivas urgentes.

Se elaboró un protocolo para la recogida de datos en el que se incluyeron la filiación, el hábitat (rural o urbano), el lugar de residencia, el estado civil, el número de medicamentos previos, el de sistemas afectados de forma crónica, el número de ingresos en el último año, la patología que motivó el ingreso y los días de hospitalización. Se recogieron tres medidas del índice de Barthel (IB); previo (dos semanas antes del ingreso), al ingreso y al alta.

Se excluyeron a los pacientes en cuatro circunstancias, según el modelo planteado por Formiga y cols. (7); aquellos dados de alta antes de 72 horas, los ingresados para recibir tratamiento paliativo, los que presentaban índice de Barthel 0 previo y los que fallecieron durante el ingreso.

Los datos se recogieron mediante entrevista con el paciente y el cuidador principal, que según el lugar de residencia era un miembro de la familia o un cuidador de la residencia asistida.

Se calculó el porcentaje de pérdida funcional respecto al estado previo mediante el cociente entre los puntos de índice de Barthel perdidos al alta respecto a la situación previa dividido entre el índice de Barthel previo.

Se utilizó el programa SPSS 10 para el análisis estadístico. Se realizó un análisis descriptivo de las variables incluidas en el estudio. Posteriormente se analizaron los valores del IB recogidos en los tres momentos mediante test no paramétricos.

Se recogieron 266 pacientes, de los cuales se excluyeron 60 $(22,6 \%)$. De ellos $35(58,3 \%)$ presentaban un IB=0 previo, $13(21,7 \%)$ fallecieron durante el ingreso, $10(16,7 \%)$ fueron dados de alta en menos de 72 horas y $2(3,3 \%)$ ingresaron para recibir tratamiento paliativo. Finalmente se incluyeron 206 pacientes. Todos provenían de los servicios de urgencias.

Los datos referentes a la edad, sexo, hábitat, residencia, estado civil, estancia media y medidas del índice de Barthel se recogen en la Tabla I.

Los pacientes presentaban una media de 2 (1-4) sistemas afectados de forma crónica, seguían tratamiento habitual con 5 (0-13) fármacos, y habían precisado 1,5 (1-4) ingresos en los doce meses previos. Los datos referentes a los motivos principales de ingreso se recogen en la Tabla II.

\section{TABLA I}

CARACTERÍSTICAS DE LOS PACIENTES INCLUIDOS EN EL ESTUDIO

\begin{tabular}{llr}
\hline Datos & \multicolumn{2}{l}{ Pacientes } \\
\hline Edad (años) & $88(80-98)$ & \\
Sexo & Varones: & $93(45,2 \%)$ \\
& Mujeres: & $113(54,8 \%)$ \\
Hábitat & Rural & $154(74,8 \%)$ \\
& Urbano & $22(25,2 \%)$ \\
Residencia & Familiar & $177(85,9 \%)$ \\
& Asistida & $29(14,1 \%)$ \\
Estado civil & Soltero & $10(4,8 \%)$ \\
& Casado & $58(28,2 \%)$ \\
& Viudo & $138(67 \%)$ \\
Estancia media (días) & $8(4-30)$ & \\
Barthel & Previo & $* 70,9(5-100)$ \\
& Ingreso & $* 48,9(0-100)$ \\
& Alta & $* 58,6(0-100)$ \\
\hline
\end{tabular}

${ }^{*} \mathrm{p}<0,001$ (comparación de las 3 medidas del índice de Barthel)
TABLA II

MOTIVOS DE INGRESO MÁS FRECUENTES

\begin{tabular}{lc}
\hline Motivo de ingreso & $N^{\circ}$ de pacientes \\
\hline $\begin{array}{l}\text { EPOC* reagudizado por Infección } \\
\quad \text { respiratoria }\end{array}$ & $44(21,4 \%)$ \\
Insuficiencia cardiaca & $40(19,4 \%)$ \\
Infección respiratoria en pacientes & \\
$\quad$ no EPOC* & $23(11,2 \%)$ \\
Neumonía & $24(11,7 \%)$ \\
Accidente cerebrovascular & $22(10,6 \%)$ \\
Gastroenteritis aguda & $7(3,4 \%)$ \\
Cardiopatía isquémica & $7(3,4 \%)$ \\
Otros & $39(18,9 \%)$ \\
\hline
\end{tabular}

*EPOC: enfermedad pulmonar obstructiva crónica.

Los pacientes que fueron exitus durante el ingreso presentaban un IB previo y al ingreso significativamente menor que el resto de la muestra con una $\mathrm{p}<0,02$ y $\mathrm{p}<0,001$ respectivamente.

Los 206 pacientes recogidos presentaban un IB previo de 70,9, al ingreso de 48,9 y al alta de 58,6, las diferencias entre las tres medidas fueron estadísticamente significativas $(\mathrm{p}<0,001)$.

$\mathrm{El}$ porcentaje de pérdida funcional obtenido en toda la muestra fue de $17,3 \%$. El destino al alta coincide con el lugar de residencia al ingreso en todos los pacientes.

\section{DISCUSIÓN}

En el presente trabajo, hemos valorado el deterioro de la capacidad funcional en pacientes ancianos ingresados en una unidad de agudos de medicina interna. Para ello, hemos utilizado el índice de Barthel, una escala de capacidad funcional que constituye además un predictor de morbi-mortalidad (11).

En nuestro estudio, en general, los pacientes presentan las características del anciano frágil, tienen pluripatología, están polimedicados y en muchos casos han precisado al menos un ingreso en el año previo. De forma similar a otros estudios el motivo principal de ingreso fue la afectación respiratoria, sobre todo de etiología infecciosa, seguida de la cardiaca mayoritariamente debida a insuficiencia cardiaca $(7,12)$. En cuanto al IB, se observa un descenso estadísticamente significativo al ingreso con una leve recuperación al alta.

La pérdida de capacidad funcional, utilizando el IB, ha sido estudiada en otros trabajos, con variabilidad en la población analizada, tanto en la edad como en las patologías que motivaron el ingreso (6,7,9-12). Así, el estudio de Formiga y cols. (7) valora la capacidad funcional después de un ingreso en pacientes nonagenarios. Incluye 125 pacientes con una edad media de 92 años, con patologías médicas y quirúrgicas incluidas traumatológicas, por lo que los datos no pueden ser directamente comparados con los que presentamos. No obstante, las medias del índice de Barthel previo al ingreso era de 75, significativamente superior al nuestro, probablemente en relación con el grupo de pacientes traumatológicos. Durante el ingreso, los pacientes del trabajo de Formiga y cols. muestran una reducción del IB a 33 puntos, con recuperación al alta hasta 43 puntos. Sin embargo a pesar de la mejor situación funcional previa, el porcentaje de 
pérdida funcional respecto al previo es de $43 \%$, superior al que encontramos en nuestro caso.

Hirsch y cols. (4) estudiaron el deterioro que se producía durante el ingreso en pacientes ancianos en la movilidad, traslado, aseo, comida, arreglo personal, continencia de esfínteres y estado mental. Excluyeron del estudio a los pacientes con accidentes cerebrovasculares. Objetivaron que en el $65 \%$ de los casos suelen presentar un deterioro significativo, sobre todo en la movilidad, al segundo día del ingreso.

Sepúlveda y cols. (12) presentan los datos de 93 pacientes nonagenarios ingresados en una unidad geriátrica de agudos. La edad media es de 92,8 años, y todos los pacientes presentan patología médica. En el artículo de Sepúlveda y cols., el IB previo es de 60 , al ingreso 23 y al alta 47 . Un $48 \%$ de los pacientes mantenían al alta el IB previo y el porcentaje de pérdida funcional respecto al previo era de $22 \%$. Sepúlveda y cols. atribuyen este mejor resultado respecto al de Formiga y cols. (7) por atender pacientes nonagenarios en unidades específicas de intervención.

El IB también se ha utilizado en otros estudios para evaluar el deterioro de la capacidad funcional en pacientes mayores de 65 años, independientes previamente para las ABVD (6,9), ingresados en unidades de corta (10) o media estancia (11). Aunque los resultados no son equiparables ya que el diseño y la metodología son distintos, todos ellos coinciden en señalar un deterioro en la capacidad funcional al ingreso con una leve recuperación al alta.

La cuestión fundamental es que podemos hacer para que nuestros pacientes tengan la menor pérdida de capacidad funcional posible. Se han realizado múltiples estudios para intentar encontrar factores de riesgo previos que permitan identificar a la población con mayor probabilidad de deterioro desde su llegada a urgencias. Algunos autores hablan de pérdida funcional asociada al decúbito, deterioro cognitivo previo o bajo nivel de actividad social (5). Otros no ven relación entre el diagnóstico principal y el deterioro, lo asocian con factores como el reposo en cama prolongado, la larga estancia hospitalaria o los efectos adversos iatrogénicos $(6,13,14)$. En otros estudios se habla de la importancia de realizar una valoración exhaustiva del paciente al ingreso y realizar cuidados en la nutrición, sondas, catéteres, movilización y rehabilitación precoz para mejorar la capacidad funcional del paciente al alta (14).

En resumen, podemos concluir que la hospitalización conlleva un deterioro importante en la capacidad funcional de los pacientes ancianos. Es necesaria la puesta en marcha de trabajos que sean capaces de identificar todos aquellos factores de riesgo responsables de la pérdida de capacidad funcional en los pacientes geriátricos, y así poder establecer pautas preventivas de actuación.

\section{Bibliografía}

1. Baztán et al. Índice de Barthel: instrumento válido para la valoración funcional de pacientes con enfermedad cerebrovascular. Rev Esp Geriatr y Gerontol 1993; 28: 32-40.

2. Valderrama E, Pérez del Molino J. Una visión crítica de las escalas de valoración funcional traducidas al castellano. Rev Esp Geriatr Gerontol 1997; 32: 297-306

3. Mahoney FI, Barthel DW. Functional evaluation: the Barthel index. Md State Med J 1965; 14: 61-65.

4. Hirsch C, Sommers L, Olsen A, Mullen L, Hutner C. The natural history of functional morbidity in hospitalized older patients. J Am Geriatr Gerontol 1990; 38: 1296-1303.

5. Inouye SK, Wagner DR, Acampora D, Horwitz RI, Cooney LM, Hurst $\mathrm{LD}$, et al. A predictive index for functional decline in hospitalized elderly medial patients. J Gen Intern Med 1993; 8: 645-652.

6. Gutiérrez J, Domínguez V, Solano JJ. Deterioro funcional secundario a la hospitalización por enfermedad aguda en el anciano. Análisis de la incidencia y los factores de riesgo asociados. Rev Clin Esp 1999; 199 : 418-423.

7. Formiga F, López A, Sacanella E, Jacob X, Masanés F, Vidal M. Valoración de la capacidad funcional después de un ingreso hospitalario en pacientes nonagenarios. Med Clin (Barc) 2000; 115: 695-696.

8. Von Strauss E, Fratiglioni L, Viitanen M, Forsell Y, Winblad B. Morbidity and comorbidity in relation to functional status: a community-based study of the oldest old(90+years). J Am Geriatric Soc 2000; 48: $1462-$ 1469.

9. Galeano R, Gutiérrez J, Reig C, San Cristóbal E, Solano JJ. Estudio de la incidencia de deterioro funcional secundario a la hospitalización en el anciano. Rev Esp Geriatr Gerontol 1997; 32: 69-73.

10. Formiga F, Maiques JM, Salazar A, Mascaro J, Novelli A, Rivera A, et al. Repercusión de la hospitalización en la capacidad funcional de pacientes mayores de 75 años ingresados en una unidad de corta estancia. Rev Esp Geriatr Gerontol 1999; 34: 331-335.

11. Valderrama E, Molpeceres J. Seguimiento a largo plazo de los pacientes ingresados en una unida de media estancia o convalescencia. Rev Esp Geriatr Gerontol 1998; 33: 67-72.

12. Sepúlveda D, Isaac M, Izquierdo G, Ruipérez I. Deterioro funcional en pacientes nonagenarios ingresados en hospitales de agudos. Med Clin (Barc) 2001; 116: 799.

13. Sager MA, Rudberg MA, Jalaluddin M, Franke T, Inouye S, Landefeld $\mathrm{S}$, et al. Hospital admission risk profile(HARP): identifying older patients at risk for functional decline following acute medical illness and hospitalization. J Am Geriatr Soc 1996; 44: 251-257.

14. Landefeld CS, Palmer RL, Kresevic DM, Fortinsky RH, Kowal J. A randomized trial of care in a hospital medical unit especially designed to improve the functional outcomes of acutely ill older patients. N Engl J Med 1995; 332: 1338-1344. 